\title{
STANDARDIZATION OF THE FEAR SURVEY SCHEDULE BASED UPON PATIENTS WITH DSM-III ANXIETY DISORDERS
}

\author{
PATRICIA TOMLIN, BRUCE A. THYER, GEORGE C. CUR'TIS, \\ RANDOLPH NESSE, OLIVER CAMERON and PHILIP WRIGHT
}

The University of Michigan

\begin{abstract}
Summary-The Wolpe-Lang Fear Survey Schedule was administered to 141 psychiatric outpatients who met the criteria for a primary Axis I diagnosis of one of the DSM-III anxiety disorders. Standardization data were obtained for this instrument and their clinical and research implications are discussed.
\end{abstract}

A large number of fear survey schedules have been developed for use in behavioral research and therapy (see e.g. Geer, 1965; Wolpe and Lang, 1964; Scherer and Nakamura, 1968; Braun and Reynolds, 1969; Goldberg, Yinon and Cohen, 1975; Granell de Aldaz, 1982) with the aim of assessing a patient's degree of fear to various phobic stimuli or to evaluate clinical outcomes of treatment for anxiety disorders. Although there is some limited support for the predictive validity of such self-report instruments with respect to phobic avoidance behavior (Lanyon and Manosevitz, 1966; Edelman, 1970; Lick, Sushinsky and Malow, 1977), recent reviews of the use of fear inventories emphasize the rudimentary state of the research on these measures (Tasto, 1977; Hersen, 1973; Wade, 1978).

For example, published normative data for a number of fear survey schedules is often based upon non-patient samples such as college students (see e.g. Geer, 1965; Wilson, 1967; Scherer and Nakamura, 1968; Bernstein and Allen, 1969; Rubin et al., 1969; Braun and Reynolds, 1969; Landy and Gaupp, 1971; Granell de Aldaz, 1982). The generalizability of such data to the psychiatric patients for whom these instruments were intended is open to question, and the use of such analogue samples has been extensively criticized in the behavioral literature (Bernstein and Paul, 1971; Rosen, 1975).
With the adoption of the psychiatric nomenclature found in the third edition of the Diagnostic and Statistical Manual of Mental Disorders (DSM-III), a further problem has arisen concerning normative data on fear survey schedules; namely that the normative parameters have changed (American Psychiatric Association, 1980). Some anxiety disorders found in DSM-II have been abolished (anxiety state), while new nosological categories have been invented (e.g. panic disorder, post-traumatic stress disorder) or more clearly delineated (e.g. agoraphobia with and without panic attacks).

One of the more widely employed fear survey schedules is the version developed by Wolpe and Lang (1969) consisting of 108 common fears which the patient is asked to rate in terms of their fear-evoking potential on a scale ranging from 0 (no fear) to 4 (very much fear). Published normative (Wolpe and Lang, 1969) and standardization (Fischer and Turner, 1978) data for the FSS have been based, like most of its predecessors, upon samples of college students. The present investigation was undertaken to provide standardization data for the Wolpe and Lang (1969) Fear Survey Schedule, based upon a sample of psychiatric patients who met the DSM-III criteria for one of the anxiety disorders.

\section{Subjects \\ METHOD \\ The subjects were 37 males and 104 females who were seen for diagnostic evaluation and treatment at the Anxiety}

Requests for reprints should be addressed to: Bruce A. Thyer, Box 011, APH-5, Anxiety Disorders Program, Department of Psychiatry, The University of Michigan Hospitals, Ann Arbor, MI 48109, U.S.A. 
Disorders Program of the University of Michigan Hospitals. Each patient was evaluated using the DSM-III guidelines and fulfilled the criteria for a primary Axis I diagnosis of one of the anxiety disorders. The sample consisted of 64 simple phobics (13 male), 34 patients with agoraphobia with panic attacks ( 5 male), 20 social phobics (10 male), 11 obsessive-compulsives ( 2 male) and 12 panic disorder patients $(7$ male). The men had a mean age of 36 years (S.D. $=13.3$ ) and the women had a mean age of 36 years (S.D. $=11.3$ ).

\section{Procedure}

The raw data for each subject's responses on the Fear Survey Schedule were transformed into standard (z) scores, with a constant of 3 added to each value to eliminate negative numbers, following the procedure of Fischer and Turner (1978). The data for each item on the Fear Survey Schedule are presented in Table 1, separated by sex.

Using this transformation, a response exactly equal to the mean response for that item in the patient population has a transformed standard score (TSS) of 3.00. A response

Table 1. Standardization data for the Fear Survey Schedule

\begin{tabular}{|c|c|c|c|c|c|c|c|c|c|c|}
\hline \multirow[b]{2}{*}{ FSS item } & \multicolumn{5}{|c|}{$\begin{array}{c}\text { Females } \\
\text { Item responses }\end{array}$} & \multicolumn{5}{|c|}{$\begin{array}{c}\text { Males } \\
\text { Item responses }\end{array}$} \\
\hline & 0 & 1 & 2 & 3 & 4 & 0 & 1 & 2 & 3 & 4 \\
\hline 1. Noise of vacuum cleaners & 2.7 & 4.8 & 6.9 & 8.9 & 11.0 & 2.6 & 4.9 & 7.2 & 9.4 & 11.7 \\
\hline 2. Open wounds & 2.0 & 2.9 & 3.8 & 4.7 & 5.7 & 2.0 & 2.8 & 3.7 & 4.5 & 5.3 \\
\hline 3. Being alone & 2.1 & 2.8 & 3.6 & 4.3 & 5.0 & 2.4 & 3.5 & 4.7 & 5.9 & 7.1 \\
\hline 4. Loud voices & 2.1 & 3.0 & 4.0 & 4.9 & 5.9 & 2.2 & 3.7 & 5.3 & 6.8 & 8.4 \\
\hline 5. Dead people & 1.9 & 2.6 & 3.4 & 4.1 & 4.9 & 1.7 & 2.7 & 3.7 & 4.7 & 5.7 \\
\hline 6. Speaking in public & 1.5 & 2.2 & 2.8 & 3.5 & 4.2 & 1.4 & 2.1 & 2.8 & 3.5 & 4.3 \\
\hline 7. Crossing streets & 2.5 & 3.6 & 4.7 & 5.7 & 6.8 & 2.6 & 5.2 & 7.9 & 10.6 & 13.3 \\
\hline 8. People who seem insane & 1.7 & 2.5 & 3.2 & 3.9 & 4.7 & 1.9 & 2.7 & 3.4 & 4.2 & 4.9 \\
\hline 9. Being in a strange place & 1.9 & 2.7 & 3.4 & 4.2 & 4.9 & 2.1 & 3.1 & 4.2 & 5.2 & 6.2 \\
\hline 10. Falling & 2.9 & 2.9 & 3.8 & 4.6 & 5.5 & 2.2 & 2.8 & 3.5 & 4.2 & 4.8 \\
\hline 11. Automobiles & 2.4 & 3.2 & 4.0 & 4.8 & 5.6 & 2.6 & 3.9 & 4.1 & 6.4 & 7.7 \\
\hline 12. Being teased & 2.2 & 3.1 & 4.1 & 5.1 & 6.0 & 2.2 & 3.0 & 3.9 & 4.8 & 5.7 \\
\hline 13. Dentists & 2.0 & 2.7 & 3.4 & 4.2 & 4.9 & 2.1 & 3.0 & 3.9 & 4.7 & 5.6 \\
\hline 14. Thunder & 2.3 & 3.1 & 4.0 & 4.9 & 5.8 & 2.5 & 4.6 & 6.7 & 8.8 & 10.9 \\
\hline 15, Sirens & 2.3 & 3.4 & 4.5 & 5.7 & 6.8 & 2.5 & 3.9 & 5.2 & 6.6 & 8.0 \\
\hline 16. Failure & 1.5 & 2.2 & 3.0 & 3.7 & 4.4 & 1.3 & 2.1 & 2.9 & 3.7 & 4.5 \\
\hline 17. Entering a room* & 2.1 & 2.8 & 3.6 & 4.3 & 5.1 & 1.9 & 2.8 & 3.7 & 4.6 & 5.5 \\
\hline 18. High places on land & 2.1 & 2.7 & 3.4 & 4.0 & 4.7 & 2.1 & 2.8 & 3.5 & 4.3 & 5.0 \\
\hline 19. High buildings* & 1.7 & 2.3 & 3.0 & 3.6 & 4.2 & 1.8 & 2.4 & 3.1 & 3.8 & 4.4 \\
\hline 20. Worms & 2.4 & 3.4 & 4.3 & 5.3 & 6.2 & 2.6 & 3.9 & 5.2 & 6.6 & 7.9 \\
\hline 21. Imaginary creatures & 2.6 & 4.3 & 6.1 & 7.9 & 9.6 & 2.6 & 4.4 & 6.2 & 8.0 & 9.9 \\
\hline 22. Receiving injections & 2.1 & 3.1 & 4.0 & 4.9 & 5.8 & 2.2 & 3.5 & 4.7 & 6.0 & 7.2 \\
\hline 23. Strangers & 2.1 & 3.0 & 4.0 & 4.9 & 5.8 & 2.2 & 3.5 & 4.7 & 5.9 & 7.1 \\
\hline 24. Bats & 1.9 & 2.6 & 3.3 & 4.1 & 4.8 & 2.2 & 3.2 & 4.2 & 5.2 & 6.2 \\
\hline 25. Journeys by train & 2.4 & 3.1 & 3.9 & 4.6 & 5.4 & 2.6 & 3.9 & 5.1 & 6.3 & 7.6 \\
\hline 26. Feeling angry & 2.1 & 2.8 & 3.6 & 4.4 & 5.1 & 2.1 & 3.0 & 3.9 & 4.8 & 5.6 \\
\hline 27. People in authority & 2.1 & 3.1 & 4.1 & 5.2 & 6.2 & 1.8 & 2.7 & 3.7 & 4.7 & 5.6 \\
\hline 28. Flying insects & 2.2 & 3.0 & 3.9 & 4.7 & 5.5 & 2.5 & 4.1 & 5.8 & 7.4 & 9.0 \\
\hline 29. Seeing others injected* & 2.3 & 3.2 & 4.2 & 5.1 & 6.1 & 2.3 & 3.7 & 5.0 & 6.4 & 7.8 \\
\hline 30. Sudden noises & 1.8 & 2.7 & 3.5 & 4.4 & 5.3 & 2.1 & 3.1 & 4.1 & 5.1 & 6.1 \\
\hline 31. Journeys by car & 2.2 & 2.9 & 3.6 & 4.3 & 5.0 & 2.4 & 3.5 & 4.5 & 5.6 & 6.6 \\
\hline 32. Dull weather & 2.4 & 3.4 & 4.4 & 5.4 & 6.4 & 2.6 & 3.6 & 4.6 & 5.6 & 6.6 \\
\hline 33. Crowds & 2.1 & 2.7 & 3.4 & 4.1 & 4.7 & 2.2 & 3.0 & 3.8 & 4.6 & 5.4 \\
\hline 34. Cats & 2.6 & 3.8 & 5.0 & 6.2 & 7.4 & 2.8 & 7.1 & 11.5 & 15.9 & 20.2 \\
\hline 35. Bullying* & 1.8 & 2.5 & 3.3 & 4.1 & 4.8 & 1.6 & 2.5 & 3.4 & 4.4 & 5.3 \\
\hline 36. Tough-looking people & 1.8 & 2.7 & 3.5 & 4.4 & 5.2 & 1.8 & 2.9 & 4.1 & 5.3 & 6.5 \\
\hline 37. Birds & 2.6 & 3.8 & 4.9 & 6.1 & 7.2 & 2.7 & 6.3 & 9.9 & 13.5 & 17.2 \\
\hline 38. Sight of deep water & 2.3 & 3.1 & 4.0 & 4.8 & 5.7 & 2.4 & 3.4 & 4.4 & 5.4 & 6.4 \\
\hline 39. Being watched working & 2.1 & 2.8 & 3.6 & 4.3 & 5.1 & 2.0 & 2.8 & 3.7 & 4.5 & 5.3 \\
\hline 40. Dead animals & 2.1 & 3.0 & 3.8 & 4.7 & 5.6 & 2.0 & 3.6 & 5.2 & 6.7 & 8.3 \\
\hline 41. Weapons & 1.9 & 2.6 & 3.4 & 4.2 & 5.0 & 2.2 & 3.1 & 4.0 & 4.9 & 5.8 \\
\hline 42. Dirt & 2.6 & 3.8 & 5.0 & 6.1 & 7.3 & 2.5 & 5.0 & 7.6 & 10.1 & 12.6 \\
\hline 43. Journeys by bus & 2.9 & 3.9 & 3.6 & 4.3 & 4.9 & 2.6 & 3.7 & 4.8 & 5.9 & 7.0 \\
\hline 44. Crawling insects & 2.0 & 2.8 & 3.6 & 4.5 & 5.3 & 2.4 & 3.7 & 5.1 & 6.5 & 7.8 \\
\hline 45. Seeing a fight & 1.7 & 2.5 & 3.2 & 4.0 & 4.8 & 1.6 & 2.7 & 3.8 & 4.9 & 5.9 \\
\hline 46. Ugly people & 2.5 & 3.8 & 5.1 & 6.4 & 7.8 & 2.4 & 3.6 & 4.8 & 6.0 & 7.2 \\
\hline 47. Fire & 2.0 & 2.7 & 3.5 & 5.2 & 5.0 & 2.2 & 3.1 & 4.0 & 4.9 & 5.8 \\
\hline 48. Sick people & 2.1 & 2.9 & 3.7 & 4.6 & 5.4 & 2.0 & 3.0 & 4.0 & 5.0 & 6.0 \\
\hline 49. Being criticized & 1.8 & 2.5 & 3.2 & 4.0 & 4.7 & 1.4 & 2.3 & 3.1 & 4.0 & 4.9 \\
\hline
\end{tabular}


Table 1 -continued

\begin{tabular}{|c|c|c|c|c|c|c|c|c|c|c|}
\hline \multirow[b]{2}{*}{ FSS item } & \multicolumn{5}{|c|}{$\begin{array}{l}\text { Females } \\
\text { Item responses }\end{array}$} & \multicolumn{5}{|c|}{$\begin{array}{c}\text { Males } \\
\text { Item responses }\end{array}$} \\
\hline & 0 & 1 & 2 & 3 & 4 & 0 & 1 & 2 & 3 & 4 \\
\hline 50. Strange shapes & 2.7 & 4.6 & 6.5 & 8.3 & 10.2 & 2.5 & 4.9 & 7.3 & 9.7 & 12.1 \\
\hline 51. Being touched by others & 2.5 & 3.7 & 4.9 & 6.2 & 7.4 & 2.2 & 3.5 & 4.7 & 6.0 & 7.3 \\
\hline 52. Being in an elevator & 2.2 & 2.9 & 3.6 & 4.2 & 4.9 & 2.1 & 3.0 & 3.8 & 4.6 & 5.4 \\
\hline 53. Witnessing surgery* & 1.9 & 2.6 & 3.2 & 3.9 & 4.6 & 1.9 & 2.6 & 3.3 & 4.0 & 4.7 \\
\hline 54. Angry people & 1.8 & 2.5 & 3.3 & 4.0 & 4.7 & 1.6 & 2.6 & 3.5 & 4.4 & 5.4 \\
\hline 55. Mice or rats & 1.7 & 2.5 & 3.2 & 3.9 & 4.6 & 2.3 & 3.2 & 4.1 & 5.0 & 5.9 \\
\hline 56. Human blood & 2.1 & 3.0 & 3.9 & 4.8 & 5.6 & 2.2 & 2.9 & 3.7 & 4.5 & 5.3 \\
\hline 57. Animal blood & 2.2 & 3.2 & 4.1 & 5.1 & 6.0 & 2.3 & 3.1 & 4.0 & 4.9 & 5.8 \\
\hline 58. Parting from friends & 2.0 & 2.7 & 3.5 & 4.3 & 5.0 & 2.0 & 3.0 & 4.0 & 5.0 & 6.0 \\
\hline 59. Enclosed places & 2.1 & 2.8 & 3.5 & 4.2 & 4.9 & 2.3 & 3.2 & 4.1 & 4.9 & 5.8 \\
\hline 60. Surgical operation* & 1.3 & 2.1 & 2.8 & 3.6 & 4.3 & 1.7 & 2.3 & 2.9 & 3.7 & 4.4 \\
\hline 61. Feeling rejected & 1.4 & 2.2 & 2.9 & 3.6 & 4.4 & 1.1 & 2.0 & 2.9 & 3.8 & 4.7 \\
\hline 62. Journeys by airplane & 1.8 & 2.5 & 3.1 & 3.7 & 4.3 & 2.1 & 2.8 & 3.4 & 4.0 & 4.6 \\
\hline 63. Medical odors & 2.3 & 3.3 & 4.3 & 5.2 & 6.2 & 2.3 & 3.3 & 4.2 & 5.2 & 6.2 \\
\hline 64. Feeling disapproved of & 1.7 & 2.4 & 3.2 & 3.9 & 4.6 & 1.4 & 2.2 & 3.0 & 3.8 & 4.6 \\
\hline 65. Harmless snakes & 2.1 & 2.9 & 3.6 & 4.3 & 5.0 & 2.2 & 3.1 & 4.0 & 4.9 & 5.8 \\
\hline 66. Cemeteries & 2.2 & 3.1 & 4.0 & 4.8 & 5.7 & 2.3 & 3.5 & 4.8 & 6.0 & 7.3 \\
\hline 67. Being ignored & 2.0 & 2.8 & 3.5 & 4.3 & 5.1 & 2.0 & 2.8 & 3.7 & 4.5 & 5.3 \\
\hline 68. Darkness & 2.1 & 3.0 & 3.9 & 4.8 & 5.7 & 2.4 & 3.5 & 4.6 & 5.7 & 6.8 \\
\hline 69. Premature heart beats* & 2.1 & 2.9 & 3.7 & 4.5 & 5.2 & 2.3 & 3.2 & 4.1 & 5.0 & 5.9 \\
\hline 70. Nude men & 2.4 & 3.6 & 4.8 & 6.0 & 7.2 & 2.6 & 4.8 & 6.9 & 9.1 & 11.3 \\
\hline 71. Nude women & 2.5 & 3.9 & 5.3 & 6.8 & 8.2 & 2.8 & 7.1 & 11.5 & 15.9 & 20.2 \\
\hline 72. Lightning & 2.1 & 2.8 & 3.6 & 4.4 & 5.2 & 2.4 & 3.9 & 5.2 & 6.5 & 7.9 \\
\hline 73. Doctors & 2.2 & 3.0 & 3.8 & 4.5 & 5.3 & 2.4 & 3.2 & 4.1 & 4.9 & 5.8 \\
\hline 74. Crippled people* & 2.3 & 3.3 & 4.2 & 5.2 & 6.2 & 2.1 & 3.1 & 4.1 & 5.1 & 6.1 \\
\hline 75. Making mistakes & 1.8 & 2.5 & 3.3 & 4.1 & 4.8 & 1.4 & 2.3 & 3.2 & 4.2 & 5.1 \\
\hline 76. Looking foolish & 1.6 & 2.3 & 3.1 & 3.8 & 4.5 & 1.2 & 2.1 & 3.0 & 3.8 & 4.3 \\
\hline 77. Losing control* & 1.6 & 2.2 & 2.9 & 3.5 & 4.1 & 1.3 & 2.1 & 2.8 & 3.6 & 4.3 \\
\hline 78. Fainting & 2.2 & 2.9 & 3.5 & 4.2 & 4.9 & 2.3 & 3.0 & 3.7 & 4.4 & 5.2 \\
\hline 79. Becoming nauseous & 2.2 & 3.0 & 3.7 & 4.5 & 5.2 & 2.3 & 3.0 & 3.8 & 4.6 & 5.4 \\
\hline 80. Harmless spiders & 2.2 & 3.0 & 3.8 & 4.7 & 5.5 & 2.3 & 3.8 & 5.4 & 6.9 & 8.5 \\
\hline 81. Decisions" & 2.0 & 2.8 & 3.5 & 4.3 & 5.1 & 2.0 & 2.9 & 3.7 & 4.5 & 5.3 \\
\hline 82. Sight of knives* & 2.5 & 3.7 & 5.0 & 6.2 & 7.4 & 2.3 & 3.0 & 3.8 & 4.6 & 5.4 \\
\hline 83. Being mentally ill* & 1.9 & 2.5 & 3.2 & 3.8 & 4.4 & 1.8 & 2.5 & 3.1 & 3.8 & 4.4 \\
\hline 84. Taking written tests & 2.1 & 2.9 & 3.7 & 4.5 & 5.3 & 2.1 & 2.9 & 3.6 & 4.3 & 5.0 \\
\hline 85. Member of opposite sex & 2.4 & 3.5 & 4.6 & 5.6 & 6.7 & 2.3 & 3.3 & 4.3 & 5.3 & 6.3 \\
\hline 86. Large open spaces & 2.4 & 3.1 & 3.8 & 4.5 & 5.3 & 2.7 & 3.7 & 4.7 & 5.7 & 6.8 \\
\hline 87. Dogs & 2.5 & 3.3 & 4.2 & 5.0 & 5.8 & 2.5 & 4.1 & 5.8 & 7.4 & 9.0 \\
\hline 88. Germs & 2.4 & 3.2 & 4.0 & 4.7 & 5.5 & 2.3 & 3.5 & 4.7 & 5.8 & 7.0 \\
\hline 89. Being seen unclothed & 2.0 & 2.9 & 3.7 & 4.6 & 5.4 & 2.0 & 3.1 & 4.1 & 5.2 & 6.2 \\
\hline 90. Taking medicine & 2.3 & 3.1 & 3.9 & 4.7 & 5.5 & 2.4 & 3.2 & 4.0 & 4.8 & 5.6 \\
\hline 91. Becoming sexually aroused & 2.6 & 3.8 & 5.1 & 6.3 & 7.5 & 2.5 & 5.0 & 7.6 & 10.1 & 12.6 \\
\hline 92. Being punished by God & 2.3 & 3.0 & 3.7 & 4.5 & 5.2 & 2.2 & 3.0 & 3.9 & 4.7 & 5.5 \\
\hline 93. Possible homosexuality* & 2.6 & 4.0 & 5.3 & 6.6 & 8.0 & 2.3 & 3.0 & 3.8 & 4.6 & 5.4 \\
\hline 94. Being dressed unsuitably* & 2.0 & 2.9 & 3.8 & 4.6 & 5.5 & 2.1 & 3.0 & 3.9 & 4.8 & 5.7 \\
\hline 95. Ministers or priests & 2.5 & 4.9 & 7.2 & 9.6 & 11.9 & 2.6 & 5.2 & 7.9 & 10.6 & 13.3 \\
\hline 96. Hurting others' feelings* & 1.6 & 2.4 & 3.1 & 3.8 & 4.6 & 1.4 & 2.3 & 3.1 & 3.9 & 4.7 \\
\hline 97. Kissing & 2.6 & 4.7 & 6.8 & 8.9 & 11.0 & 2.4 & 4.0 & 5.6 & 7.2 & 8.8 \\
\hline 98. Undertakers & 2.4 & 3.3 & 4.2 & 5.1 & 6.0 & 2.4 & 3.6 & 4.8 & 6.0 & 7.2 \\
\hline 99. Police & 2.4 & 3.5 & 4.6 & 5.8 & 6.9 & 2.3 & 3.9 & 5.4 & 7.0 & 8.5 \\
\hline 100. Fish & 2.7 & 4.8 & 6.9 & 8.9 & 11.0 & 2.6 & 4.8 & 6.9 & 9.1 & 11.3 \\
\hline 101. Masturbation & 2.6 & 3.7 & 4.8 & 5.9 & 7.0 & 2.4 & 3.8 & 5.2 & 6.5 & 7.9 \\
\hline 102. Leaving home & 2.1 & 2.8 & 3.4 & 4.1 & 4.7 & 2.4 & 3.5 & 4.6 & 5.7 & 6.8 \\
\hline 103. Physical examinations & 2.1 & 2.9 & 3.7 & 4.4 & 5.2 & 2.3 & 3.2 & 4.1 & 4.9 & 5.8 \\
\hline 104. Marriage & 2.3 & 3.2 & 4.1 & 5.0 & 5.9 & 2.3 & 3.3 & 4.3 & 5.3 & 6.3 \\
\hline 105. Insecticides & 2.4 & 3.5 & 4.5 & 5.5 & 6.6 & 2.4 & 3.5 & 4.5 & 5.6 & 6.7 \\
\hline 106. Vomiting & 2.3 & 3.0 & 3.7 & 4.4 & 5.1 & 2.3 & 3.1 & 3.8 & 4.6 & 5.3 \\
\hline 107. Responsibility* & 2.0 & 2.8 & 3.6 & 4.4 & 5.2 & 1.9 & 2.8 & 3.6 & 4.4 & 5.2 \\
\hline 108. Hospitals & 2.1 & 2.8 & 3.4 & 4.1 & 4.8 & 2.2 & 2.9 & 3.6 & 4.3 & 5.0 \\
\hline
\end{tabular}

Wording of item has been abridged. 
with a TSS $>3.00$ would be above the mean, while a TSS $<3.00$ would indicate a response below the mean for that patient group (male or female). The data were not separately analyzed by diagnosis due to the limited number of patients in some of the diagnostic categories. The mean total Fear Survey Schedule score based upon untransformed data was 94.5 (S.D. $=50.2$ ) for males and 113.6 (S.D. $=64.5$ ) for females. The scores ranged from 18 to 225 for men and from 8 to 294 for women.

\section{DISCUSSION}

It is important to note, as did Fischer and Turner (1978), the distinction between using the FSS ipsatively and normatively. Clinically the FSS is most useful as an ipsative instrument to assess the degree of clustering of phobic stimuli for an individual patient and as an outcome measure to evaluate the effectiveness of treatment. Normatively these data, which are presented on an interval scale as opposed to the original ordinal scale of measurement, can be employed in future research with the FSS using non-clinical or analogue phobic subjects to determine the extent to which such a sample resembles psychiatric patients. The present data also permit a clinician to compare the responses of an individual patient to a standardized group of patients with a DSM-III anxiety disorder.

\section{REFERENCES}

American Psychiatric Association (1980) Diagnostic and Statistical Manual of Mental Disorders, 3rd Edn. American Psychiatric Association, Washington D.C.

Bernstein D. and Allen G. (1969) Fear Survey Schedule (II): Normative data and factor analysis based upon a large college sample. Behav. Res. \& Ther. 7, 403-408. Bernstein D. and Paul G. (1971) Some comments on therapy analogue research with small animal "Phobias". $J$. Behav. Ther. \& Exp. Psychiat. 2, 225-237.

Braun P. and Reynolds D. (1969) A factor analysis of a 100-item fear survey inventory. Behav. Res. \& Ther. 7, 399-402.

Edclman R. (1970) Validity of verbal report as a prognosticator of physiological arousal to threat. J. $A b n$. Psychol. 76, 492-495.

Fischer S. and Turner R. (1978) Standardization of the Fear Survey Schedule. J. Behav. Ther. \& Exp. Psychiat. 9, 129-133.

Geer J. (1965) The development of a scale to measure fear. Behav. Res. \& Ther. 3, 45-53.

Goldberg J., Yinon Y. \& Cohen A. (1975) A factor analysis of the Israeli Fear Survey Inventory. Psychol. Rep. 36, 175-179.

Granell de Aldaz E. (1982) Factor analysis of a Venezuelan fear survey schedule. Behav. Res. \& Ther. 20, 313-322.

Hersen M. (1973) Self-assessment of fear. Behav. Ther. 4, 241-257.

Lanyon R. and Manosevitz M. (1966) Validity of selfreported fear. Behav. Res. \& Ther. 4, 259-263.

Landy F. and Gaupp L. (1971) A factor analysis of the Fear Survey Schedule. Behav. Res. \& Ther. 9, 89-93.

Lick J., Sushinsky L. and Malow R. (1977) Specificity of Fear Survey Schedule items and the prediction of avoidance behavior. Behav. Mod. 1, 195-203.

Rosen G. (1975) Is it really necessary to use mildly phobic analogue subjects? Behav. Ther. 6, 68-71.

Rubin S., Lawlis G., Tasto D. and Namenek T. (1969) Factor analysis of the 122 item Fear Survey Schedule. Behav. Res. \& Ther. 7, 381-386.

Scherer M. and Nakamura C. (1968) A Fear Survey Schedule for children (FSS-FC): A factor analytic comparison with manifest anxiety (CMAS). Behav. Res. \& Ther. 6, 173-182.

Tasto D. (1977) Self-report schedules and inventories. In Handbook of Behavioral Assessment (Edited by Ciminero A., Calhoun K. and Adams H.). Wiley, New York.

Wade T. (1978) Factor analytic approaches to the investigation of common fears: A critical appraisal and reanalysis. Behav. Ther. 9, 923-935.

Wilson G. (1967) Social desirability and sex differences in expressed fear. Behav. Res. \& Ther. 5, 136-137.

Wolpe J. and Lang P. (1964) A Fear Survey Schedule for use in behavior therapy. Behav. Res. \& Ther. 2, 27-30.

Wolpe J. and Lang P. (1969) Fear Survey Schedule. Educational and Industrial Testing Service, San Diego, California. 